\title{
Multiuser Detection for MIMO CDMA Systems
}

\author{
M.Angeline \\ Student, M.E., Applied Electronics, \\ Anna University Tirunelveli, \\ Tirunelveli
}

\author{
S.Lenty Stuwart \\ Lecturer, Department of ECE, \\ Anna University Tirunelveli, \\ Tirunelveli.
}

\begin{abstract}
MIMO is a technique to increase data rate significantly with multiple antennas at both the transmitter and receiver. MIMO takes the advantage of random fading and multipath delay spread. MIMO systems will need to function reliably in interference limited environment in order to be effective. CDMA systems are designed to operate in an interference free environment and for this reason it is used in modern cellular systems. The combination of MIMO and CDMA can further improve the system transmission rate over the traditional CDMA system. Multiuser MIMO CDMA systems are considered where each user has multiple transmit antennas, different transmit antennas of the same user use the same spreading code. Matched filter method and decorrelating detector method are used to detect the signals with Gaussian Noise. In many wireless systems the ambient noise is known through experimental measurements to be decidedly non-Gaussian due to largely impulsive phenomena. The performance of many multiuser detectors can degrade substantially in the presence of such impulsive ambient noise. For combating Multi Access Interference and impulsive noise in CDMA communication systems, a technique based on $\mathrm{m}$ estimation is used. Performance comparison shows that $\mathrm{m}$ estimation has better performance under non-Gaussian noise than the other detection techniques.
\end{abstract}

\section{Keywords}

Spreading, Fading, Noise, Linear Detection, Nonlinear Detection

\section{INTRODUCTION}

The developing wireless services require higher data rates from future cellular wireless communication systems. However, new radio frequency bands are very scarce if available at all. CDMA is a promising technique for beyond $3 \mathrm{G}$ wireless systems. The main technical and theoretical challenges in future wireless system concepts creation are:

1) Bandwidth efficiency challenge $(2-10 \mathrm{~b} / \mathrm{s} / \mathrm{Hz})$

2) Frequency selectivity challenge due to the large bandwidth $(\approx 100 \mathrm{MHz})$

The bandwidth efficiency challenge requires novel solutions in both the network and physical layers. The latter could include powerful coding and modulation methods, transmission adaptation techniques, and antenna configurations. Multiple-input multiple-output (MIMO) communications based on multiple transmit and receive antennae is a very promising technique to increase bandwidth efficiency, and is seen as a potential key solution for fading channels with rich enough scattering. The frequency selectivity challenge means that the multipath delay

spread of the channel is very large due to the large bandwidth, causing very severe intersymbol interference (ISI) [11].

\begin{abstract}
Multiple-input and multiple-output (MIMO) is a technique to increase data rate significantly with multiple antennas at both the transmitter and receiver. In MIMO systems, there are many spatial coding schemes including space time codes with spatial code rate less than or equal to 1 [19] and spatial multiplexing with spatial code rate > 1 [18], [20]. By combining MIMO and CDMA, the resulting MIMO CDMA system [4],[5] can further improve the system transmission rate over the traditional CDMA system. In the MIMO CDMA systems considered in the literature, there are two different approaches to assign spreading codes. The one is multiple spreading code approach, in which different antennas are assigned to different spreading codes such as [1], [4],[10]. Since the data streams of different antennas are distinguishable by different spreading codes, the inter antenna interference (IAI) is greatly reduced. The other is single spreading code approach, in which different antennas are assigned to the same spreading code [4]. In this case, the error rate performance is dictated by IAI [11]. Deng et al. [6] propose a detection method with single spreading code approach in the V-BLAST coded DS-CDMA system. This scheme uses a redundant bit (spatial code rate $=$ the number of transmit antenna for one user/2) in order to identify the transmit antenna. This coding scheme results in data rate loss. The performance of non Gaussian noise is improved using differential detection and L-D (Limiter Discriminator) detection. L-D detector outperforms differential detection. The signal detectors designed for Gaussian statistics may suffer significant degradation when the actual statistics deviate from the Gaussian model [21]. Both man-made noise and low frequency atmospheric interference environments are basically impulsive noise. That is they have a highly structured form, characterized by significant probabilities of large interference level. Under such environment the performance of the receiver systems, designed to perform optimally in Gaussian noise, degrades significantly. For this a non linear decorrelating detector is used to improve the performance of the system.

This paper is organized as follows: The system model is described in Section II. Section III describes the results. The conclusion is given in Section VI.
\end{abstract}

\section{SYSTEM DEFINITION}

$K$-user uplink CDMA system is considered. Each user (mobile station) has $N t$ transmit antennas and the base station has $\mathrm{Nr}$ receive antennas. For simplicity, PAM modulation is used. The extension to higher-order modulation formats is straightforward but at the cost of higher complexity.

\subsection{Transmitter}

At the transmitter, data streams are passed through serial to parallel converter, modulation, and spreading. The structure of the transmitter is depicted in Fig. 1. At the $m$-th symbol interval [ $(m-$ 
1)T,mT], the data symbol $d(m) k, i$ is transmitted through the $i$-th transmit antenna of the $k$-th user, $i=1, \ldots, N t, k=1, \ldots, K$, where $N t$ denotes the number of the transmit antenna. $T$ denotes the symbol interval and $m$ denotes the symbol index, $m=1,2, \ldots, M$, where $M$ is the number of symbols in one data frame. Then, the data symbol is spread by the aperiodic spreading sequence (periodic spreading sequence is its special case) shown in equation (1). (see Next Page). Result is shown for both msequence and orthogonal codes.

\section{First user Transmitter}

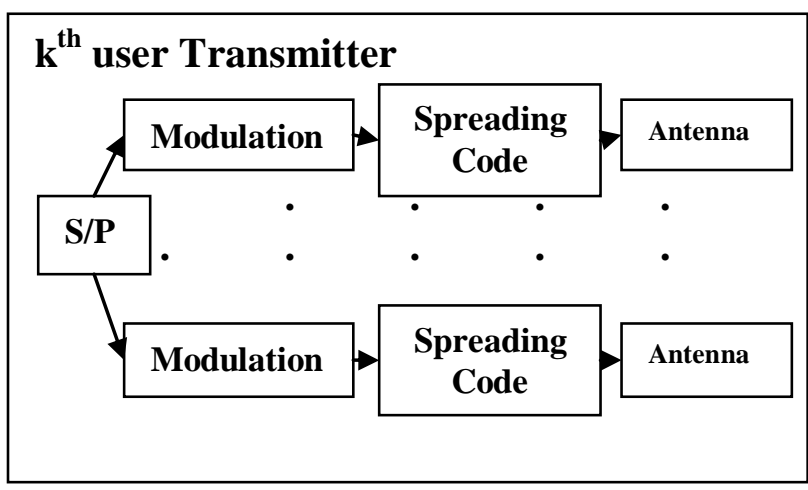

\section{Kth user Transmitter}

Figure 1: Structure of Transmitter

$N$ is the processing gain, $c(m-1) N+n, k, \quad n=0,$. . ., $N-1$, is the spreading sequence for the user $k$, and $\psi(t)$ is the normalized chip waveform with duration $[0, T c]$. The spreading sequence $s k(t)$ is independent of the transmit antenna index $i$, because we use the same spreading sequence in all transmit antennas of one user. In (1), the scalar $1 / \sqrt{N}$ makes each user's transmitted power be independent of the number of the transmit antenna $(N t)$. The equation of signal transmission for the $k$-th user's $i$-th transmit antenna is given by

$$
x_{k, i}(t)=\sum_{m=1}^{m} d_{k}, i(m) s_{k}(t-(m-1) T), \quad i=1,2, \ldots . . N_{t}
$$

\subsection{Multipath fading Channel}

The channel coefficients can be calculated according to the formula [7]

$$
\begin{gathered}
c(k)=-a_{1} c(k-1)-a_{2} c(k-2)+w(k) \\
a_{1}=-2 r_{d} \cos \left(2 \pi f_{p} \mathbf{T}\right) \\
a_{2}=r_{d}^{2}
\end{gathered}
$$

$$
f_{p}=f_{d} \sqrt{2}
$$

Where $\mathrm{w}(\mathrm{k})$ - complex zero mean white Gaussian process, $\mathrm{a}_{1}, \mathrm{a}_{2}$ - Physical Parameter, $\mathrm{f}_{\mathrm{p}}$ - spectral peak frequency, $\mathrm{r}_{\mathrm{d}}$-pole radius. Let $L$ denote the number of multiple paths. $\tau_{k, j, l}^{(m)}$ denotes the $l$-th multipath delay from the $k$-th user's transmit antennas to the $j$-th receive antenna at the $m$-th symbol interval. $h_{k, i, j, l}^{(m)}$ denotes the $l$ th multipath channel coefficient from the $k$-th user's $i$-th transmit antenna to the $j$-th receive antenna at the $m$-th symbol interval. These $L$ multipaths from one transmit antenna to one receive antenna are correlated. The channel is a time-varying correlated multipath fading channel with normalized Doppler frequency $f d$

$$
f_{d}=\frac{V \max f_{c}}{c}
$$

where $V \max , f c$, and $c$ denote maximum velocity of the mobile unit, radio carrier frequency, and speed of light, respectively. The channel coefficients are

$$
h_{k, i, j}^{(m)}=\left[h_{k, i, j, 1}^{(m)} \ldots . . h_{k, i, j, L}^{(m)}\right]
$$

\subsection{Noise Function}

Noise is added to the faded signal in the channel. Error rate is calculated for various SNR values. Real time noise contains manmade noise and some low frequency atmospheric interference. They follow non Gaussian distribution. Non Gaussian noise is designed using the following formula [2], [12], [21]

$$
f=(1-\xi) N\left(0, v^{2}\right)+\xi N\left(0, k v^{2}\right)
$$

With $v>0,0 \leq \xi \leq 1, k \geq 1$. The $N\left(0, v^{2}\right)$ term represents the nominal background noise, and the $N\left(0, k v^{2}\right)$ term represents an impulsive component, with $\xi$ representing the probability that impulses occur. The total noise variance is given by

$$
\sigma^{2} \triangleq(1-\xi) v^{2}+\xi k v^{2}
$$

\subsection{Receiver}

The received signal includes all of the transmitted signals multiplied by an $L$-path Rayleigh fading process from all transmit antennas and the noise in the receive antennas. Thus, the received signal at the $j$-th receive antenna has the lowpass equivalent representation given by (4). At the $m$-th symbol interval, $k$-th user matched filter output is sampled at $t=m T+\tau_{k, i, l}^{(m)}, l=1,2, \ldots . ., L \quad(L$ multiple paths $)$ to form the statistics. 


$$
\begin{gathered}
S_{k}(t-(m-1) T)=\sum_{n=0}^{N-1} \frac{1}{\sqrt{N_{t} N}} c_{(m-1) N+n, k} \psi\left(t-(m-1) T-n T_{c}\right) \\
r_{j}(t)=\sum_{m=1}^{M} \sum_{k=1}^{K} \sum_{i=1}^{N t} \sum_{l=1}^{L} d_{k, j}^{(m)} s_{k}\left(t-(m-1) T-\tau_{k, j, l}^{(m)}\right) h_{k, i, j, l}^{(m)}+n_{j}(t)
\end{gathered}
$$

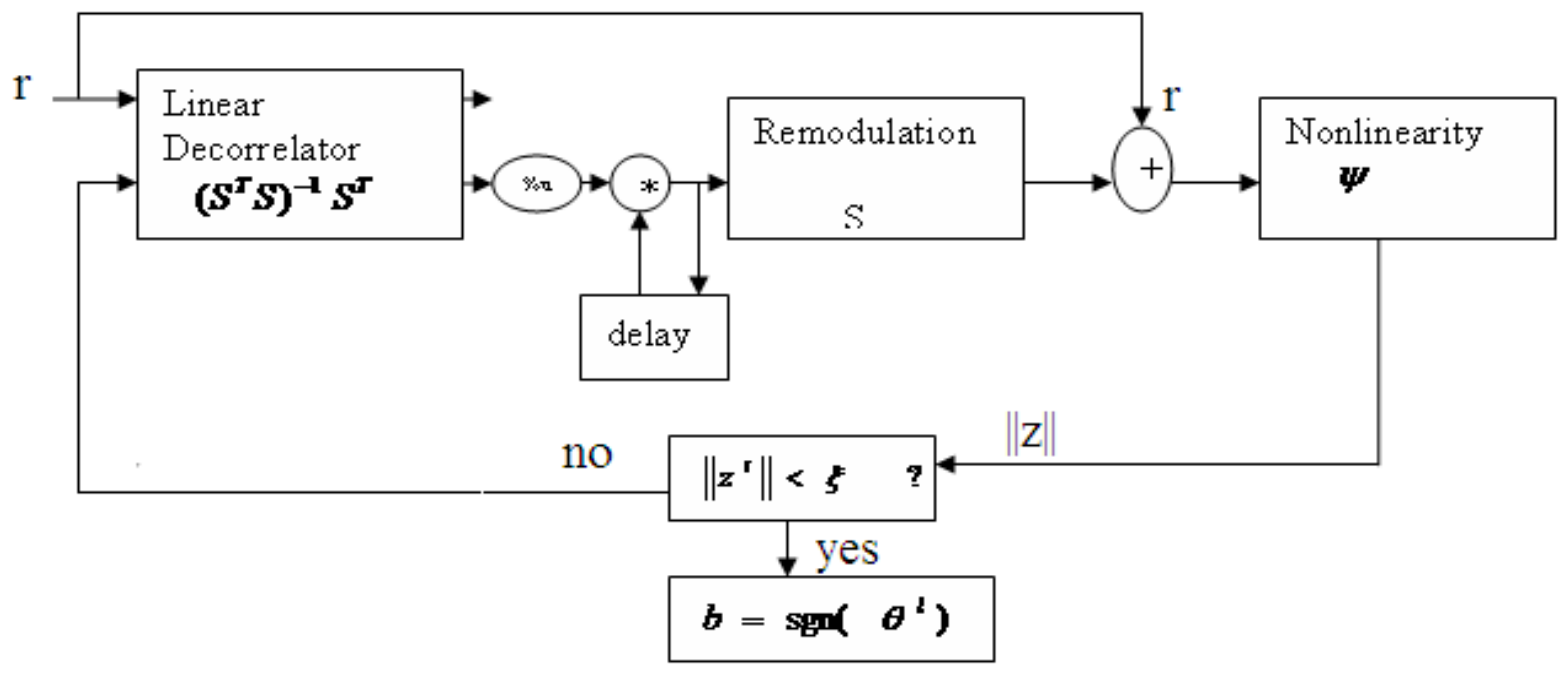

Fig.3. Block Diagram of m-estimation Method

Matched filter method and decorrelating detection method are used to detect the signals.

\subsubsection{Matched Filter Method}

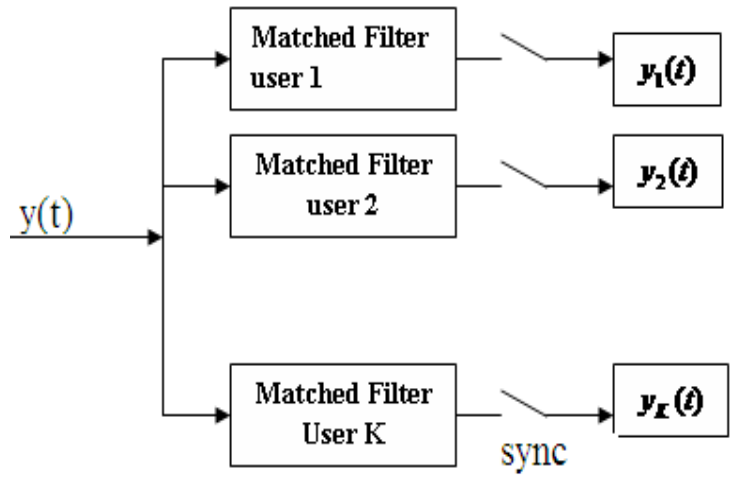

Figure 2: Block Diagram of Matched Filter

$$
=\sum_{i=1}^{N_{t}} \boldsymbol{d}_{k, i}^{(m)} \boldsymbol{h}_{k, i, j}^{(m)}+\overline{\boldsymbol{n}}_{k, j}^{(m)}
$$

Where $y_{k, j, l}^{m}$ is the matched filter output and $\overline{n_{k, j}^{(m)}}$ contains filtered noise.

\subsubsection{Decorrelating Detector}

The received signal is despread with the PN sequence, and then multiplied with the covariance matrix $\mathrm{R}^{-1}$. They are given by the following equations.

$$
\begin{aligned}
r e c & =r * S \\
R & =S * S^{T} \\
o p & =\operatorname{rec} * \operatorname{inv}\left(S * S^{T}\right)
\end{aligned}
$$

where $\mathrm{S}$ denotes the spread sequence of all the users

$$
y_{k, j}^{(m)}=\left[y_{k, j, 1}^{(m)}, \ldots \ldots \ldots y_{k, j, L}^{(m)}\right]^{T}
$$




\subsubsection{M-Estimation Method}

Performance of the system under non-Gaussian noise is poor compared to that of the Gaussian noise. M-estimation method is used to improve the performance of the system. Linear detectors do not perform well under non-Gaussian noise. Non-linear detectors (Huber Estimator) are used under such cases, even though they are complex to design. ( Fig. 3.See Previous page)

$$
\begin{gathered}
z^{l}=\psi\left(r-S^{T} \theta^{l}\right) \\
\theta^{l+1}=\theta^{l}+\frac{1}{\mu}\left(S^{T} S\right)^{-1} S^{T} z^{l} \\
\psi(x)= \begin{cases}\frac{x}{\sigma^{2}}, & \text { for }|x| \leq k \sigma^{2} \\
k \operatorname{sgn}(x), & \text { for }|x|>k \sigma^{2}\end{cases}
\end{gathered}
$$

Where $\quad \theta=\left(S^{T} S\right)^{-1} S^{T} r$

$$
\begin{aligned}
& \theta=R^{-1} S^{T} r \\
& k=\frac{1.5}{\sigma}
\end{aligned}
$$

$\mu$ is the step parameter, $\mu=\sigma^{2}, \psi$ is the non linear function

\section{RESULTS AND DISCUSSION}

Performance of the MIMO CDMA systems with Gaussian and ambient impulsive non-Gaussian noise are analyzed with orthogonal codes and non-orthogonal codes, msequence. The received signal is processed through either matched filter or decorrelating detector. Performance of the system under non-Gaussian noise is poor compared to that of Gaussian noise. M-estimation method is used to improve the performance of the system under non-Gaussian noise. Two user two antenna systems are considered. Input sequence eof length 100000 is used. Outputs are shown for m-sequence spreading and orthogonal code spreading. For orthogonal codes a sequence of length 4 is used. For m-sequence the sequence length is taken to be 7. Performance of the system under non-Gaussian noise is poor compared to that of the Gaussian noise, for both the spreading sequences, Fig (4), (5), (7), (8). Output of the mestimation for Gaussian and non-Gaussian noise is shown in Fig (6) and (9). BER of the non-Gaussian noise is reduced compared to that of the Gaussian noise. Table shows the BER for user 1 for all the SNR values between 15 and 30 . The following outputs depict the performance comparison.

\subsection{For Orthogonal codes}

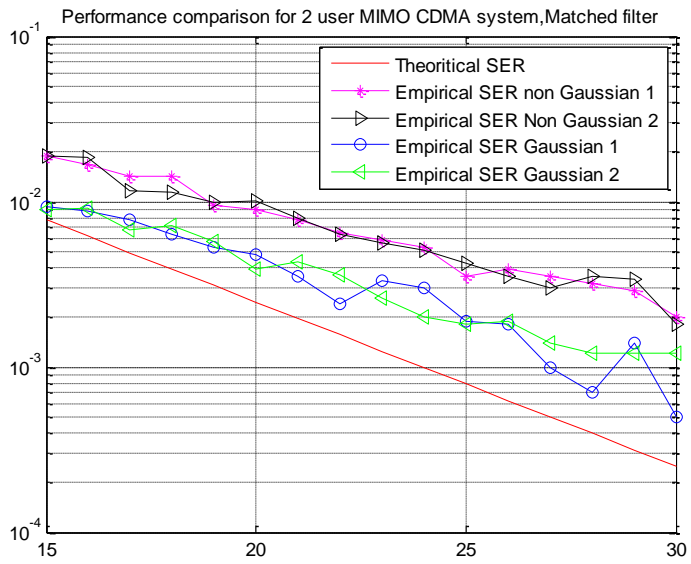

Fig .4. Output for Matched Filter

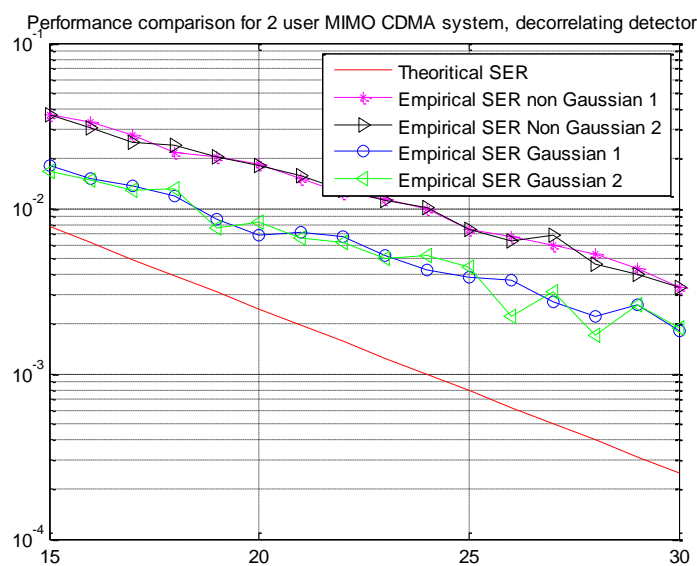

Fig.5. output for Decorrelating Detector

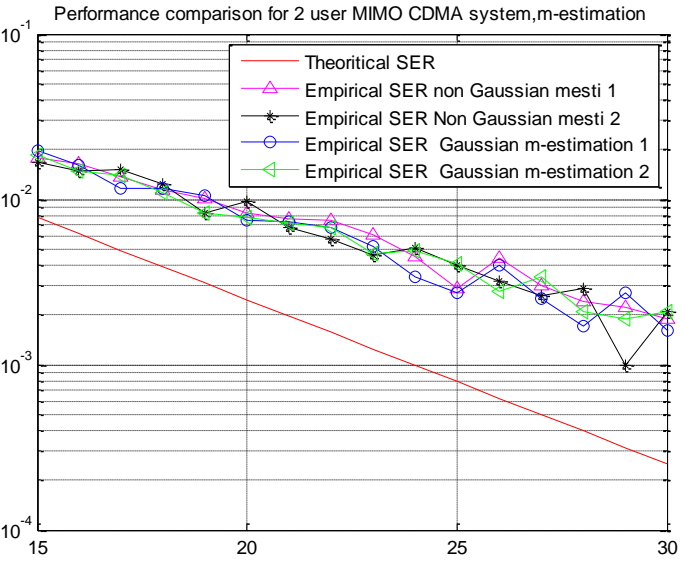

Fig.6. Output for M-estimation 


\subsection{For M-sequence}

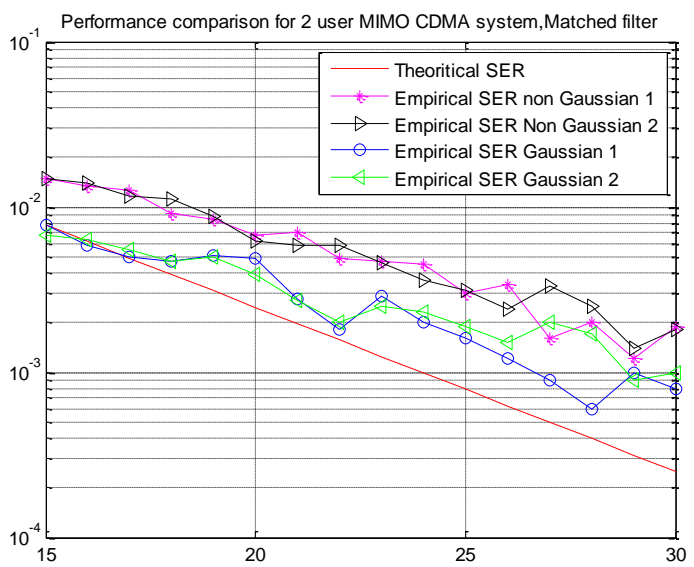

Fig .7. Output for Matched Filter

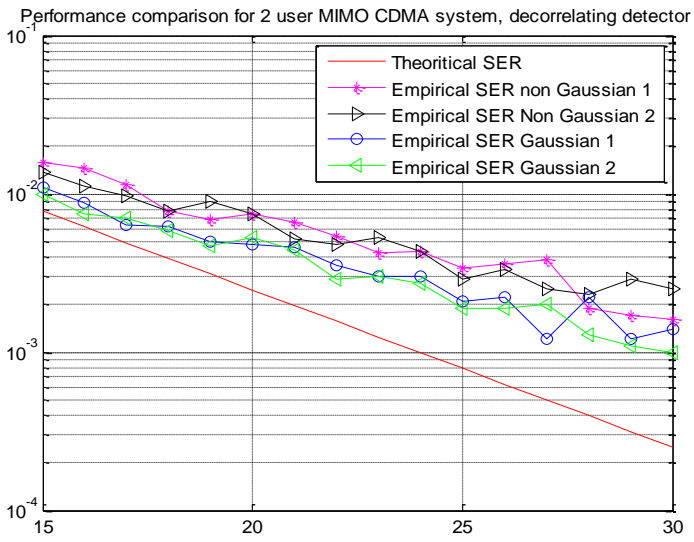

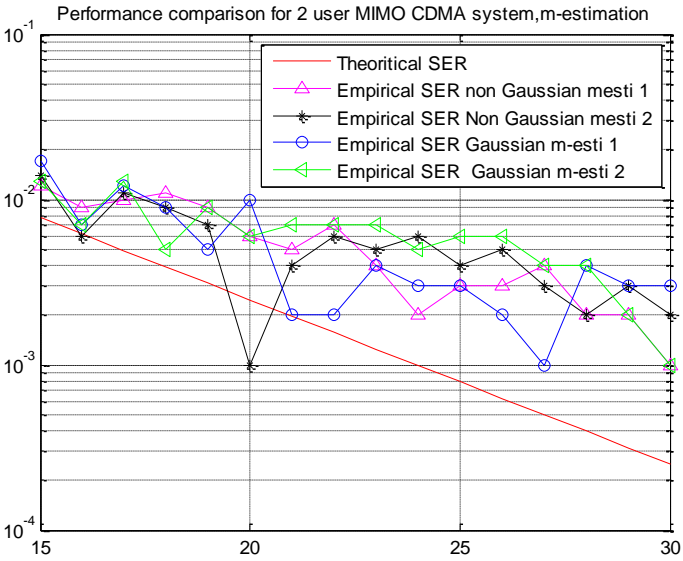

Fig.9. Output for M-estimation

Table shows the performance Comparison for different estimators. Theoretical value is compared with the practical value. Signal is degraded by fading and noise while it travels along the channel. Nonlinear decorrelating detectors perform better in the presence of ambient noise. From the table we can infer the performance improvements.

Fig.8. output for Decorrelating Detector

Table.1. Performance comparison for different estimators with Orthogonal Spreading, (MF-Matched Filter, DD-Decorrelating Detector)

\begin{tabular}{|l|l|l|l|l|l|l|l|}
\hline \multicolumn{2}{|l|}{} & \multicolumn{2}{|c|}{ MF } & \multicolumn{2}{c|}{ DD } & \multicolumn{2}{c|}{ M-estimation } \\
\hline SNR & $\begin{array}{l}\text { Theoretical } \\
\text { BER }\end{array}$ & $\begin{array}{l}\text { Gaussian } \\
\text { Noise }\end{array}$ & $\begin{array}{l}\text { Non- Gaussian } \\
\text { Noise }\end{array}$ & $\begin{array}{l}\text { Gaussian } \\
\text { Noise }\end{array}$ & $\begin{array}{l}\text { Non-Gaussian } \\
\text { Noise }\end{array}$ & $\begin{array}{l}\text { Gaussian } \\
\text { Noise }\end{array}$ & $\begin{array}{l}\text { Non-Gaussian } \\
\text { Noise }\end{array}$ \\
\hline 15 & 0.0077 & 0.0093 & 0.0189 & 0.0181 & 0.0371 & 0.0197 & 0.0178 \\
\hline 16 & 0.0062 & 0.0087 & 0.0169 & 0.0151 & 0.0335 & 0.0160 & 0.0165 \\
\hline 17 & 0.0049 & 0.0078 & 0.0142 & 0.0136 & 0.0279 & 0.0116 & 0.0137 \\
\hline 18 & 0.0039 & 0.0063 & 0.0141 & 0.0118 & 0.0216 & 0.0116 & 0.0113 \\
\hline 19 & 0.0031 & 0.0053 & 0.0095 & 0.0086 & 0.0203 & 0.0105 & 0.0102 \\
\hline 20 & 0.0025 & 0.0048 & 0.0090 & 0.0069 & 0.0186 & 0.0074 & 0.0082 \\
\hline 21 & 0.0020 & 0.0035 & 0.0078 & 0.0072 & 0.0152 & 0.0073 & 0.0076 \\
\hline 22 & 0.0016 & 0.0024 & 0.0065 & 0.0068 & 0.0123 & 0.0067 & 0.0074 \\
\hline 23 & 0.0012 & 0.0033 & 0.0058 & 0.0052 & 0.0113 & 0.0052 & 0.0061 \\
\hline 24 & 0.0010 & 0.0030 & 0.0053 & 0.0042 & 0.0099 & 0.0034 & 0.0045 \\
\hline 25 & 0.0008 & 0.0019 & 0.0035 & 0.0038 & 0.0074 & 0.0027 & 0.0029 \\
\hline 26 & 0.0006 & 0.0018 & 0.0039 & 0.0037 & 0.0068 & 0.0040 & 0.0044 \\
\hline 27 & 0.0005 & 0.0010 & 0.0035 & 0.0027 & 0.0060 & 0.0025 & 0.0030 \\
\hline 28 & 0.0004 & 0.0007 & 0.0032 & 0.0022 & 0.0053 & 0.0017 & 0.0024 \\
\hline 29 & 0.0003 & 0.0014 & 0.0029 & 0.0026 & 0.0043 & 0.0027 & 0.0022 \\
\hline 30 & 0.0002 & 0.0005 & 0.0020 & 0.0018 & 0.0033 & 0.0016 & 0.0019 \\
\hline
\end{tabular}


Table.2. Performance comparison for different estimators M-Sequence Spreading

(MF-Matched Filter, DD-Decorrelating Detector)

\begin{tabular}{|l|l|l|l|l|l|l|l|}
\hline \multicolumn{2}{|l|}{} & \multicolumn{2}{|c|}{ MF } & \multicolumn{2}{c|}{ M-estimation } \\
\hline SNR & $\begin{array}{l}\text { Theoretical } \\
\text { BER }\end{array}$ & $\begin{array}{l}\text { Gaussian } \\
\text { Noise }\end{array}$ & $\begin{array}{l}\text { Non- } \\
\text { Gaussian } \\
\text { Noise }\end{array}$ & $\begin{array}{l}\text { Gaussian } \\
\text { Noise }\end{array}$ & $\begin{array}{l}\text { Non } \\
\text { Gaussian } \\
\text { Noise }\end{array}$ & $\begin{array}{l}\text { Gaussian } \\
\text { Noise }\end{array}$ & $\begin{array}{l}\text { Non- } \\
\text { Gaussian } \\
\text { Noise }\end{array}$ \\
\hline 15 & 0.0077 & 0.0078 & 0.0148 & 0.0110 & 0.0158 & 0.0170 & 0.0120 \\
\hline 16 & 0.0062 & 0.0058 & 0.0135 & 0.0087 & 0.0146 & 0.0070 & 0.0090 \\
\hline 17 & 0.0049 & 0.0050 & 0.0127 & 0.0064 & 0.0113 & 0.0120 & 0.0100 \\
\hline 18 & 0.0039 & 0.0047 & 0.0091 & 0.0062 & 0.0077 & 0.0090 & 0.0110 \\
\hline 19 & 0.0031 & 0.0051 & 0.0085 & 0.0050 & 0.0069 & 0.0050 & 0.0090 \\
\hline 20 & 0.0025 & 0.0049 & 0.0068 & 0.0048 & 0.0075 & 0.0100 & 0.0060 \\
\hline 21 & 0.0020 & 0.0028 & 0.0070 & 0.0046 & 0.0066 & 0.0020 & 0.0050 \\
\hline 22 & 0.0016 & 0.0018 & 0.0049 & 0.0035 & 0.0054 & 0.0020 & 0.0070 \\
\hline 23 & 0.0012 & 0.0029 & 0.0047 & 0.0030 & 0.0042 & 0.0040 & 0.0040 \\
\hline 24 & 0.0010 & 0.0020 & 0.0045 & 0.0030 & 0.0043 & 0.0030 & 0.0020 \\
\hline 25 & 0.0008 & 0.0016 & 0.0030 & 0.0021 & 0.0034 & 0.0030 & 0.0030 \\
\hline 26 & 0.0006 & 0.0012 & 0.0034 & 0.0022 & 0.0036 & 0.0020 & 0.0030 \\
\hline 27 & 0.0005 & 0.0009 & 0.0016 & 0.0012 & 0.0038 & 0.0010 & 0.0040 \\
\hline 28 & 0.0004 & 0.0006 & 0.0020 & 0.0022 & 0.0019 & 0.0040 & 0.0020 \\
\hline 29 & 0.0003 & 0.0010 & 0.0012 & 0.0012 & 0.0017 & 0.0030 & 0.0020 \\
\hline 30 & 0.0002 & 0.0008 & 0.0019 & 0.0014 & 0.0016 & 0.0030 & 0.0010 \\
\hline
\end{tabular}

BER for Gaussian noise and Non-Gaussian noise for Matched Filter, Decorrelating Detector and M- Estimation method is shown. It can be inferred that the performance of M-estimation method under Non-Gaussian noise is better compared to that of the Gaussian noise.

\section{CONCLUSION}

In many practical wireless channels in which multiuser detection techniques may be applied, the ambient noise is likely to have an impulsive component that gives rise to larger tail probabilities than is predicted by the Gaussian model. Impulsive noise can seriously degrade the error probability of the linear multiuser detectors for the given level of noise power. Mestimation method is used to improve the performance of the system under non-Gaussian noise. Performance of the system under Gaussian and non-Gaussian noise is shown in the table for Matched filter and Decorrelating detector for two user two antenna systems. Theoretical value is compared with the practical value. M-estimation method gives better performance under non-Gaussian noise compared to that of the Matched filter and decorrelating detector.

\section{REFERENCES}

[1] Shu-Ming Tseng , (2009)“ Sequential Detection for MIMO CDMA system with single spreading code per user" IEEE tarnsaction on Wireless Communications, vol.8, No.7.

[2] T.Anil Kumar, (2009) "A Robust Multiuser Detection based scheme for cross talk mitigation in DMT VDSL with Non Gaussian Noise", Electronics ang Communication Engg. Dept. kakatiya Institute of Technology and Science, Warangal, India.
[3] A. Bhargave, R. J. P. de Figueiredo, and T. Eltoft, "A detection algorithm for the V-BLAST system," in Proc. IEEE Globecom, Nov. 2001, vol. 1, pp. 494-498.

[4] H.-H. Chen and M. Guizani, "Multiple access technologies for $\mathrm{B} 3 \mathrm{G}$ wireless communications," IEEE Commun. Mag., vol. 43, no. 2, pp. 65-67, Feb. 2005.

[5] W. Choi and J. Andrews, "On spatial multiplexing in cellular MIMOCDMA systems with linear receiver," in Proc. IEEE ICC, 2005, vol. 2, pp. 1337-1342.

[6] K. Deng, Q. Yin, L. Ding, and Z. Zhao, "Blind channel estimator for V-BLAST coded DS-CDMA system in frequency-selective fading environment," in Proc. IEEE VTC 2003-Fall, Oct. 2003, vol. 1, pp. 458-462.

[7] P. Dent, G. E. Bottomley, and T. Croft, "Jakes fading model revisited," IEEE Electron. Lett., vol. 29, no. 13, pp.1162-1163, June 1993.

[8] G. J. Foschini and M. J. Gans, "On the limits of wireless communication in a fading environment when using multiple antennas," IEEE Wireless Personal Commun., vol. 6, no. 3, pp. 311-335, 1998

[9] W. Gao, S. Tsai, and J. S. Lehnert, "Diversity combining for DS/SS systems with time-varying, correlated fading branches," IEEE Trans. Commun., vol. 51, no. 2, pp. 284-295, Feb. 2003.

[10] D. Gesbert, M. Shafi, D. S. Shiu, P. J. Smith, and A. Naguib, "From theory to practice: an overview of MIMO space-time coded wireless systems," IEEE $J$. 
Select. Areas Commun., vol. 21, no. 3, pp. 281-

302, Apr. 2003.

[11] M. Juntti et al., "MIMO MC-CDMA communications for future cellular system," IEEE Commun. Mag., vol. 43, no. 2, pp. 118-124, Feb. 2005.

[12] Nazh Guney, Hakan Delic, and Mutlu Koca, "Robust Multiuser detection for Impulse Radio in Non-Gaussian UWB Channels", wireless communication Laboratory, Dept of EEE, Bogazici University, Turkey

[13] A. Nordio and G. Taricco, "Linear receivers for the multiple-input multiple-output multiple-access channel," IEEE Trans. Commun., vol. 54, no. 8, pp. 14461456, Aug. 2006.

[14] K.N. Plataniotis, A.N.Venetsanopoulos, (2000), "State Estimation in the presence of NonGaussian noise", Department of ECE, University of Toronto.

[15] D. Reynolds, X. Wang, and H. V. Poor, "Blind adaptive space-time multiuser detection with multiple transmitter and receive antennas," IEEE Trans. Signal Processing, vol. 50, no. 6, pp. 1261-1276, June 2002.

[16] M. K. Simon and M. S. Alouini, Digital Communications Over Fading Channels: A Unified Approach Performance Analysis. New York: Wiley, 2000.

[17] S. M. Tseng, H. C. Yu, C. L. Chang, Y. C. Tseng, and K. C. Lin, "EM vector channel estimation and V-BLAST/PIC detection for multiuser long-code MIMO CDMA in multipath rayleigh fading channels," in Proc. IEEE AINA, Mar. 2005, vol. 1, pp. 565-569.

[18] S.-M. Tseng and H.-L. Lee, "An adaptive partial parallel multistage detection for MIMO systems," IEEE Trans. Commun., vol. 53, no. 4, pp. 587-591, Apr. 2005.

[19] Tarokh, et al., "Space-time codes for high data rate wireless communications: performance criterion and code construction," IEEE Trans. Inform. Theory, vol. 44, no. 2, pp. 744-765, Mar. 1998.

[20] P. W. Wolniansky, G. J. Foschini, G. D. Golden, and R. A. Valenzuela, "V-BLAST: an architecture for realizing very high data-rates over the rich-scattering wireless channel," in Proc. IEEE ISSSE, Sept. 1998, pp. 295300 .

[21] Xiaodong wang, (1999) "Robust Multiuser detection in Non Gaussian channels" IEEE transaction on signal processing, vol.47, NO.2,

[22] P. H.-Y. Wu and A. Duel-Hallen, "Multiuser detectors with disjoint Kalman channel estimators for synchronous CDMA mobile radio channels," IEEE Trans. Commun., vol. 48, no. 5, pp. 752-756, May 2000.

[23] Zartash Afzal Uzmi (2002) "Simplified Multiuser Detection for CDMA Systems", a dissertation submitted to the Department of Electrical Engineering and the committee on graduate studies of Stanford University 